\title{
Design of rural energy systems
}

\author{
SIDDHARTHA BHATT M \\ Central Power Research Institute, Bangalore 560012, India
}

MS received 9 July 1982; revised 28 May 1984

\begin{abstract}
Current focus on rural energy has resulted in the emergence of numerous strategies and a variety of technologies employing differentconversion processes and sources. This paper suggests a methodology for finding out the overall first law system efficiency for various routes of energy supply. The method is not an optimisation technique but gives clues regarding the choice of energy sources for maximum overall efficiency of the system. Results indicate that a decentralised system with multi-energy sources is the most efficient (efficiency $12 \%$ ) for villages.
\end{abstract}

Keywords. Decentralised energy; centralised energy; combined system; system efficiency.

\section{Introduction}

The three important elements in an energy system are the source, the task and the conversion device. Efficient energy conversion demands that these three be matched in all respects.

There are four main technical problems which limit the overall efficiency of an energy system, viz energy losses through: (i) devices of low overall efficiency (I law), (ii) improper matching of sources and tasks (II law inefficiencies), (iii) adoption of long energy conversion chain routes involving unnecessary energy vectors (steam, compressed air, etc) when shorter routes are available, (iv) long transportation routes when shorter routes or the possibility of elimination of transportation exist.

To overcome these a choice of the most efficient energy system must be made within cost constraints. Rural energy, being a major sector, has been selected for study.

The paper aims at examining the different routes of rural energy conversion with respect to the I law efficiency, transportation routes and energy conversion chains. II law analysis and economic analysis have not been attempted.

\section{Energy systems}

Modern approaches to the rural energy problem are based on one of the three concepts - the centralised system, the decentralised system and the combined system.

The centralised energy system consists of a nucleus or a few energy nuclei where large amounts of energy of the order of megawatts are generated and transported to wide areas around, eg, a coal mine, an oil well, a thermal power plant, a hydroelectric generator, a nuclear reactor and so on. The energy is converted into a 'useful form' like upgraded coal, petroleum oil or electricity, and then transported to the load points.

The decentralised system is characterised by the absence of the large capacity and the 
intricate distribution network found in the large energy systems. The systems are mostly renewable and consist of small sources of the order of kilowatts or watts (eg, animal powered irrigation, village wood lots, solar photovoltaics, etc) which energise local tasks especially in villages. The small capacity of decentralised systems permit variety and flexibility in the choice of an appropriate system. The elimination of distribution and transportation enable a direct and sometimes thermodynamically better energy conversion route.

In practice, the major fraction of rural energy is of the decentralized type with minor centralised inputs. Such a 'real system' which integrates both concepts is called a combined energy system.

\section{System terminology}

For the sake of convenience, the decentralised systems are described first, the centralised systems next and the combined systems last. The following common terminology is used for all systems.

System: A village of known population.

Source: The source is the starting point in the energy conversion chain inside the system. Its energy value is called the source end energy. Examples of sources are firewood, agrowastes, electricity at load point, direct solar radiation, etc.

External energy source: In the centralised system, the energy in its final state prior to transportation/transmission is taken as the external energy source, eg, electricity at the generating point, diesel or kerosene at the refinery, coal at the processing plant, etc. The external energy differs from the source end energy by the transportation/transmission efficiency from the generating point to the system boundaries.

Resource: The source of the centralised system at the starting point is called the resource, for example, coal in mines, crude oil in wells, etc. The energy at this point is called the resource end energy.

Secondary energy inputs: In centralised systems, in the production of an energy form, secondary inputs are needed. To produce electricity in a coal thermal plant, inputs such as diesel for firing the boilers and for transportation of coal, human energy in the power plant, etc, are required. Similarly in the transportation of diesel, coal is needed; in the transportation of firewood, coal, human energy and animal energy, etc, are needed. These are referred to as secondary energy inputs.

Task: A task is an energy-consuming activity at the end of the conversion chain. The main aim is to provide energy for various tasks.

\section{General assumptions}

\subsection{Resource end, source end and task end energies}

Values of energy consumption at the source end and at the task end cannot be added to each other because of the efficiency factors involved between the two. Similarly resource and task end energies are connected by the efficiency factors. Energies have to be added 
at common points such as the source or the task end because their values change all along the conversion chain by virtue of the various device conversion efficiencies.

\section{2 "Free" and "exerted" energy forms}

Sunshine, flow of water, wind, etc, in their natural form are considered free energy inputs and their contributions are not considered in calculations. The contributions of exerted energies like animal power, diesel, wood, etc, which involve expenditure or effort for their harnessing are taken into account.

However, if free rural energies are harnessed by solar collectors, water turbines, windmills, etc, then their contribution becomes valid.

\subsection{Sources, tasks and common energy}

There are $p$ sources (except in the one source model) and $q$ tasks. The source end energies are $S_{k}$ (where $k=1$ to $p$ ) and end point energies are $T_{e}$ (where $e=1$ to $q$ ). The external energies are $E_{k}(k=1$ to $p)$ and the resource energies are $R_{k}(k=1$ to $p)$. The common energy sources are electricity or biogas.

\section{Mathematical formulations of the different models}

\subsection{Decentralised energy' systems}

5.1 a Common energy mode: In this mode, all sources are used to produce a common intermediate energy form like electricity or biogas. The common energy is supplied to all the rural loads under consideration.

(i) One source model: This model assumes a single energy source $(S)$ which is converted into electricity or biogas $(E)$.

The single source may be firewood for electricity (or animal dung for biogas). Generation of $E$ from $S$ involves $n$ energy conversions each of overall efficiency $\eta_{i}(i=1$ to $n)$. The utilisation of $E$ for a task $T_{l}$ involves $m$ conversions each of overall efficiency $\eta_{j l}(j=1$ to $m$ ). Figure 1 gives the source-task diagram of the one source, common energy decentralized system.

The overall efficiency of the route $S \rightarrow E \rightarrow T_{l}$ is

$$
\eta_{o l}=\left(\prod_{i=1}^{n} \eta_{i}\right)\left(\prod_{j=1}^{m} \eta_{j l}\right) .
$$

The source end energy for a task $T_{l}$ is

$$
S_{l}=T_{l}\left(\eta_{o l}\right)^{-1} \text {. }
$$

The total source end energy for $q$ tasks is

$$
S=\left[\sum_{l=1}^{q}\left(\eta_{o l}\right)^{-1} T_{l}\right] .
$$

The overall system efficiency is

$$
\eta_{o \text { system }}=\left(\sum T_{l}\right)(S)^{-1}
$$




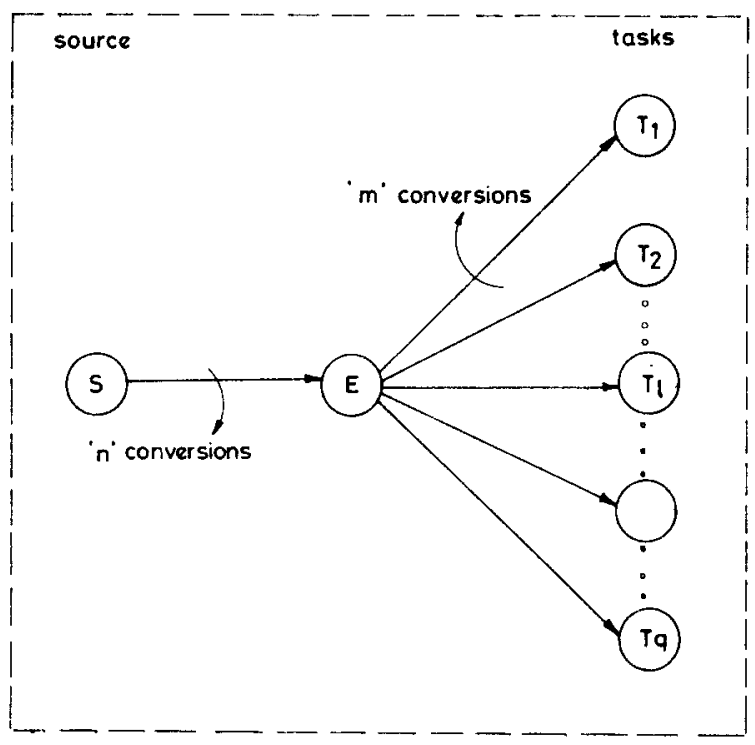

Figure 1. Source-task diagram of the one source-common energy-decentralized system (S - source energy, $E$-common energy, $T_{e}$ - task energy)

(ii) Multi-source model: This model assumes that electricity is generated by numerous sources. Generation of $E$ from a source $S_{-k}$ involves $n$ conversions each of overall efficiency $\eta_{i k}(i=1$ to $n)$. The utilisation of $E$ for a task $T_{l}$ involves $m$ conversions each of overall efficiency $\eta_{j l}(j=1$ to $\mathrm{m})$.

Figure 2 gives the source-task diagram of the multi-source, common energy decentralised system.

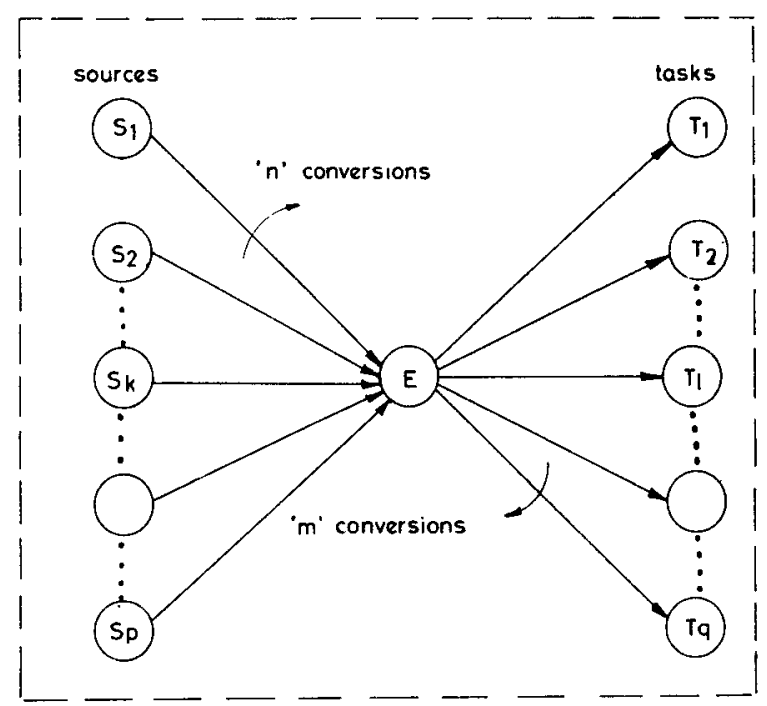

Figure 2. Source-task diagram of the multi-source-common energy-decentralized system $\left(S_{k}\right.$ - source energy, $E$ - common energy, $T_{e}$-task energy) 
The $E$ generated from a source $S_{k}$ is

$$
E_{k}=\left[\left(\prod_{i=1}^{n} \eta_{i k}\right) S_{k}\right]
$$

The $E$ utilised for a task $T_{l}$ is

$$
E_{l}=\left[\prod_{j=1}^{m}\left(\eta_{j l}\right)^{-1} T_{l}\right]
$$

Conservation of energy demands that

i.e.,

$$
\left(\sum_{k=1}^{p} E_{k}\right)=\left(\sum_{l=1}^{q} E_{l}\right)
$$

$$
\sum_{k=1}^{p} S_{k}\left(\prod_{i=1}^{n} \eta_{i k}\right)=\left[\sum_{l=1}^{q} T_{l}\left(\prod_{j=1}^{m} \eta_{j l}\right)^{-1}\right] .
$$

The overall system efficiency is

$$
\eta_{o \text { system }}=\left(\sum_{l=1}^{a} T_{l}\right)\left(\sum_{k=1}^{p} S_{k}\right)^{-1} .
$$

$5.1 b$ One energy mode: In this mode, a single source provides energy for all tasks without the intermediate conversion into a 'common energy'. The utilisation of $S$ for task $T_{l}$ involves $n$ conversions each of overall efficiency $\eta_{i l}(i=1$ to $n)$.

Figure 3 gives the source-task diagram of the one source decentralised system.

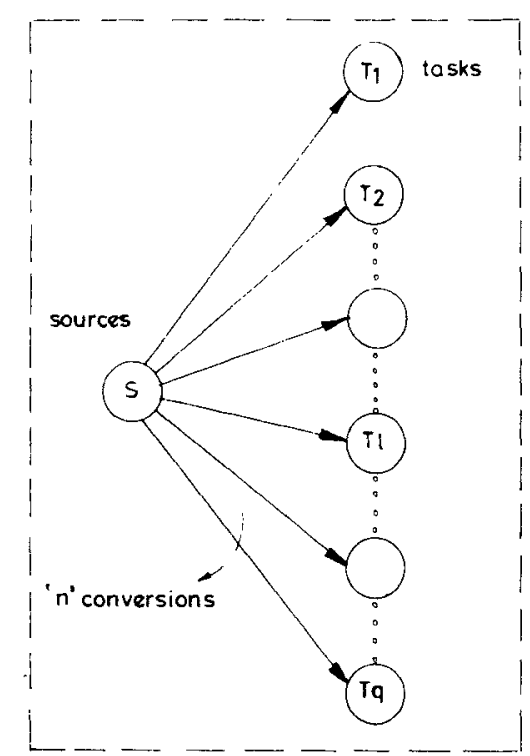

Figure 3. Source-task diagram of the one-source-decentralized system ( $\mathrm{S}$-source energy, $T_{\mathrm{e}}$-task energy) 
The overall efficiency of route $S \rightarrow T_{1}$ is

$$
\eta_{a i}=\left(\prod_{i=1}^{n} \eta_{i t}\right)
$$

The source end energy for task $T_{l}$ is

$$
S_{l}=\left[\left(\eta_{o l}\right)^{-1} T_{l}\right]
$$

The total source end energy for $q$ tasks is

$$
S=\left[\sum_{l=1}^{q}\left(\eta_{o l}\right)^{-1} T_{l}\right] .
$$

The overall system efficiency is

$$
\eta_{o \text { system }}=\left(\sum T_{l}\right)(S)^{-1}
$$

5.1c Multi-energy mode: In this model, the tasks are performed by sources that match it technologically (biogas for cooking, solar electricity for lighting, firewood by producer gas route for pumping, etc). Thermodynamically there may exist a better match but appropriate technology for such energy conversion may not be available.

It is assumed that utilisation of a fraction $\left(S_{k l}\right)$ of a source $S_{k}$ to perform a fraction $\left(T_{k l}\right)$ of a task $T_{e}$ involves $n$ conversions each of overall efficiency $\eta_{i k l}(i=1$ to $n)$. Figure 4 gives the source-task diagram of the multi-source decentralized system.

The task end energy fraction of task $T_{l}$ derived from a source $k$ is

$$
T_{k l}=S_{k l}\left(\prod_{i=1}^{n} \eta_{i k l}\right) .
$$

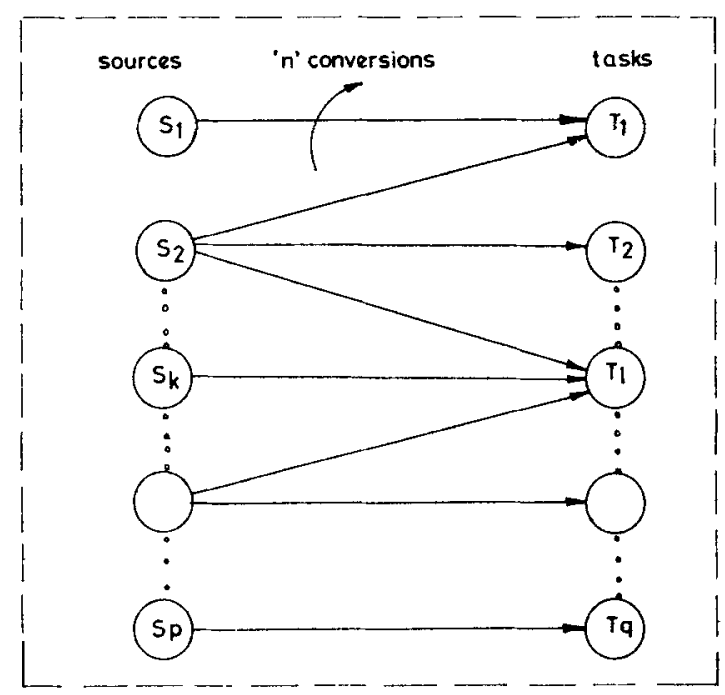

Figure 4. Source-task diagram of the multi-source-decentralized system $\left(S_{k}-\right.$ source energy, $T_{e}$ - task energy) 
The total source end energy is

$$
\sum_{l=1}^{q} \sum_{k=1}^{p} S_{k l}=\left[\sum_{l=1}^{q} \sum_{k=1}^{p} T_{k l}\left(\prod_{i=1}^{n} \eta_{i k l}\right)^{-1}\right] .
$$

The overall system efficiency is

$$
\eta_{o \text { system }}=\frac{\sum_{l=1}^{q}\left(T_{l}\right)}{\sum_{k=1}^{p}\left(S_{k}\right)}=\frac{\sum_{l=1}^{q}\left(\sum_{k=1}^{p} T_{k l}\right)}{\sum_{k=1}^{p}\left(\sum_{l=1}^{q} S_{k l}\right)} .
$$

5.1d Complex energy mode: This combines the multiple source model of the common energy mode and the multi-energy mode. Some electricity is generated from different sources and used for multiple tasks like pumping, lighting and rural industry. Along with this firewood is used for cooking, etc.

The tasks are separated into electrically energised tasks $T_{l x}\left(l x=1\right.$ to $\left.q_{x}\right)$ and nonelectrical tasks $T_{l y}\left(l y=1\right.$ to $\left.q_{y}\right)$. Correspondingly the energy sources are subdivided into electricity producing sources $S_{k x}\left(k x=1\right.$ to $\left.p_{x}\right)$ and non-electricity producing sources, $S_{k y}\left(k y=1\right.$ to $\left.p_{y}\right)$. To simplify the situation, the system is so chosen that conversion of $S_{k x}$ to $T_{l y}$ and $S_{k y}$ into $T_{l x}$ is not possible. In the electrical system (suffix $x$ ), it is assumed that the conversion of source $S_{k x}$ into electricity $E_{k x}$ involves $n$ conversions each of overall efficiency $\eta_{i_{1} k x}\left(i_{1}=1\right.$ to $\left.n\right)$. The utilisation of electricity $E_{l x}$ for a task $T_{l x}$ involves $m$ conversions each of overall efficiency $\eta_{i_{2} l x}\left(i_{2}=1\right.$ to $\mathrm{m}$ ). In the non-electrical system (suffix $y$ ), it is assumed that the utilisation of a fraction $\left(S_{k l y}\right)$ of source $S_{k y}$ to perform a fraction $\left(T_{k l y}\right)$ of a task $\left(T_{l y}\right)$ involves $n$ conversions each of overall efficiency $\eta_{i k l y}(i=1$ to $n$ ).

Figure 5 gives the source-task diagram of the complex energy mode, decentralised system.

Conversion of energy at the electricity production point demands that

$$
\sum_{k x=1}^{p x} S_{k x}\left(\prod_{i_{1}=1}^{n} \eta_{i_{2} k x}\right)=\sum_{l x=1}^{q x} T_{l x}\left(\prod_{i_{2}=1}^{m} \eta_{i_{2} l x}\right)^{-1} .
$$

The total non-electrical source end energy is

$$
\sum_{k y=1}^{p y} \sum_{l y=1}^{q y} S_{k l y}=\left[\sum_{k y=1}^{p y} \sum_{l y=1}^{q y} T_{k l y}\left(\prod_{i=1}^{n} \eta_{i k l y}\right)\right]^{-1} .
$$

The overall system efficiency is

$$
\begin{aligned}
\eta_{o \text { system }}= & \frac{\sum_{l=1}^{q} T_{l}}{\sum_{k=1}^{p} S_{k}}=\frac{\left(\sum_{l x=1}^{q x} T_{l x}\right)+\left(\sum_{l y=1}^{q y} T_{l y}\right)}{\left(\sum_{k x=1}^{p x} S_{k x}\right)+\left(\sum_{k y=1}^{p y} S_{k y}\right)} \\
& =\frac{\left[\sum_{l x=1}^{q x} T_{l x}\right]+\left[\sum_{l y=1}^{q y}\left(\sum_{k y=1}^{p y} T_{k l y}\right)\right]}{\left[\sum_{k x=1}^{p x} S_{k x}\right]+\left[\sum_{k y=1}^{p y}\left(\sum_{l y=1}^{q y} S_{k l y}\right)\right]}
\end{aligned}
$$




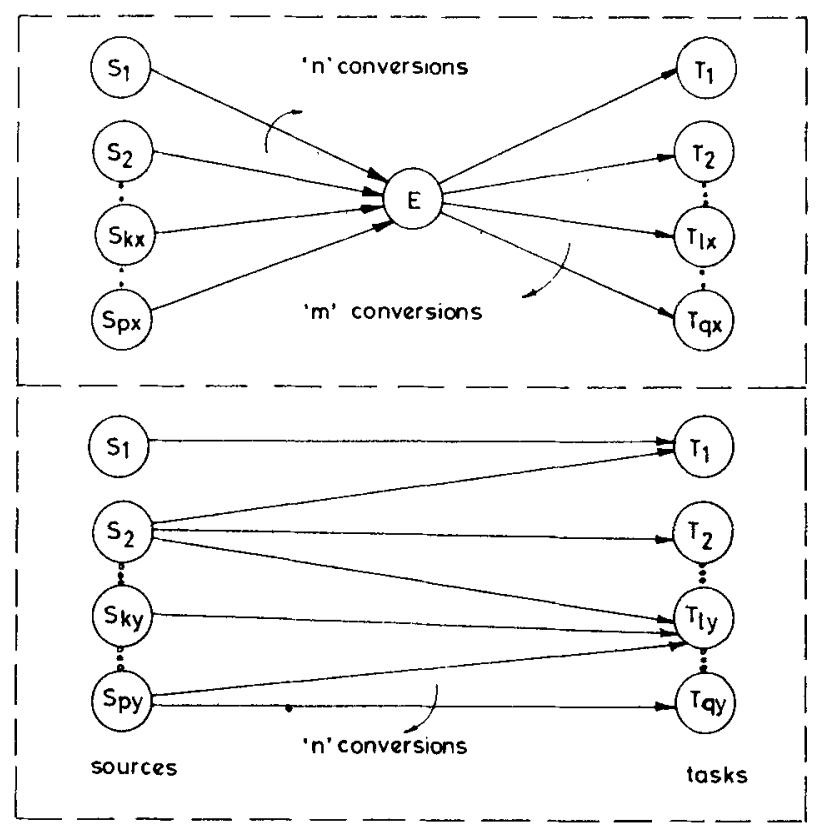

Figure 5. Source-task diagram of the complex-energy mode-decentralized system $\left(S_{k}\right.$-source energy, $E$-common energy, $T_{e}$ - -task energy)

\subsection{Centralised energy system}

5.2a Non-secondary inputs: In this model, secondary inputs associated with the external energy sources are not considered.

A look at figures 1 to 8 indicates that systems described by figures 1 to 5 are closed with respect to import or export of energy. Hence, their overall system efficiency is the ratio of the task end energy to the source end energy. The systems described by figures 6 to 8 import energy from outside. In these cases two types of efficiencies can be considered.

(i) A system efficiency which is the ratio of the task end energy and the source end energy irrespective of the resource system. If electricity is available to the system from a thermal power plant and this is stepped down inside the system $(98 \%$ efficiency), transmitted at a low voltage ( $96 \%$ efficiency) and used for a motor ( $70 \%$ efficiency) which runs a centrifugal pumpset (60\% efficiency), the system efficiency for this subsystem is $98 \times 0.96 \times 0.70 \times 0.60=39.51 \%$.

(ii) An overall system efficiency which considers the efficiency of the resource system. This is the ratio of the task end energy to the resource end energy. If the electricity from the above sub-system is obtained from coal with the following efficiencies: collection$90 \%$, transport and purification- $-90 \%$, steam power plant $-30 \%$, transmission and distribution till the village entry $-90 \%$, then the overall system efficiency is $90 \times 0.90$ $\times 0.30 \times 0.90 \times 0.3951=8.64 \%$.

Thus in the centralised and combined systems both system and overall system efficiencies have to be considered to get a clear picture and to know if the loss is in the production or the transit stage or inside the system during conversion. 
The following assumptions are made for the centralised energy system:

(i) Conversion of resource energy $R_{k}$ to external energy source $E_{k}$ involves $n_{3}$ conversions each of overall efficiency $\eta_{i 3 k}\left(i_{3}=1\right.$ to $\left.n_{3}\right)$.

(ii) Conversion of $E_{k}$ to $S_{k}$ involves $m_{1}$ inputs each of magnitude $F_{j l}\left(j_{l}=1\right.$ to $\left.m_{1}\right)$. conversions each of overall efficiency $\eta_{i_{2} k}\left(i_{2}=1\right.$ to $\left.n_{2}\right)$.

(iii) Utilisation of a fraction $\left(S_{k l}\right)$ of a source $S_{k}$ to perform a fraction $\left(T_{k l}\right)$ of a task $T_{l}$ involves $n_{1}$ conversions each of overall efficiency $\eta_{i, k l}\left(i_{1}=1\right.$ to $\left.n_{1}\right)$.

Figure 6 gives the resource-task diagram of the centralised energy system with no secondary inputs.

The total source end energy is

$$
\begin{aligned}
& \sum_{l=1}^{q} \sum_{k=1}^{p} S_{k l}=\sum_{l=1}^{q} \sum_{k=1}^{p} T_{k l}\left(\prod_{i_{1}=1}^{n_{1}} \eta_{i_{1} k l}\right)^{-1}, \\
& \sum_{k=1}^{p} \sum_{l=1}^{q} S_{k l}=\sum_{k=1}^{p} S_{k}=\sum_{k=1}^{p}\left(\prod_{i_{2}=1}^{n_{2}} \eta_{i_{2} k}\right) E_{k}, \\
& \sum_{k=1}^{p} E_{k}=\sum_{k=1}^{p}\left(\prod_{l_{3}=1}^{n_{3}} \eta_{i_{3} k}\right) R_{k} .
\end{aligned}
$$

The total resource energy consumption is

$$
\sum_{k=1}^{p} R_{k}=\sum_{k=1}^{p}\left[\left(S_{k}\right)\left(\prod_{i_{2}=1}^{n_{2}} \eta_{i_{2} k}\right)^{-1}\left(\prod_{i_{3}=1}^{n_{3}} \eta_{i_{3} k}\right)^{-1}\right]
$$

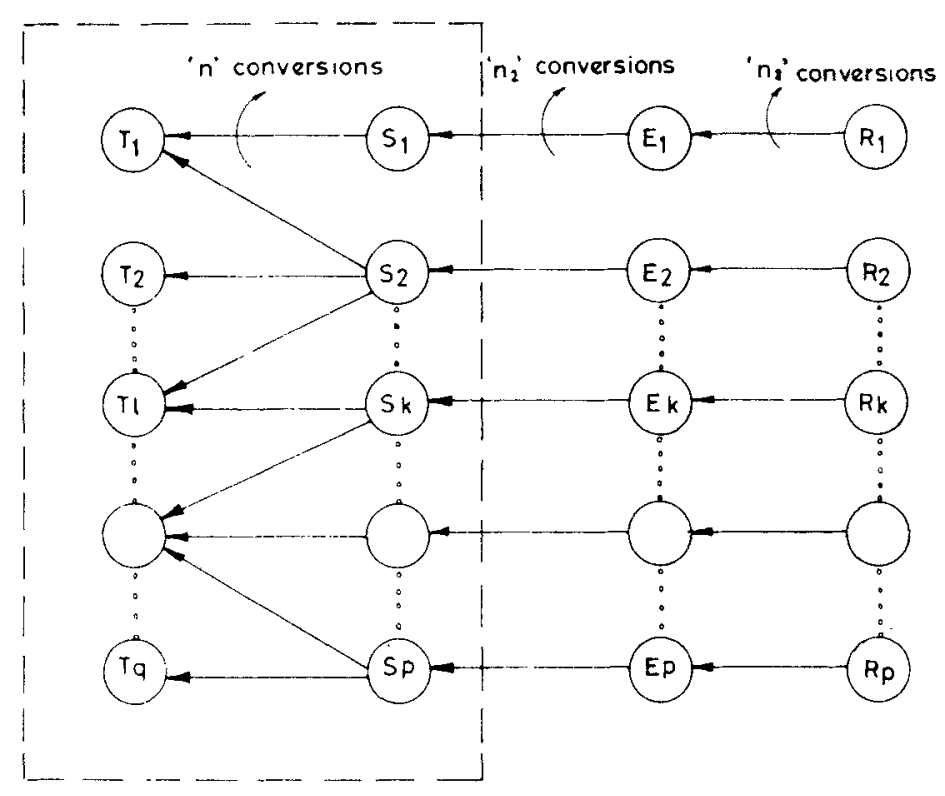

Figure 6. Resource-task diagram of the centralized energy system with no secondary inputs $\left(T_{e}\right.$ - task energy, $S_{k}$-source energy, $E_{k}$-intermediate energy, $R_{k}$-resource energy) 
The system efficiency is

$$
\eta_{\text {system }}=\left[\sum_{l=1}^{q} T_{l}\right]\left[\sum_{k=1}^{p} S_{k}\right]^{-1} .
$$

The overall system efficiency is

$$
\eta_{\text {osystem }}=\left[\sum_{l=1}^{q} T_{l}\right]\left[\sum_{k=1}^{p} R_{k}\right]^{-1} .
$$

$5.2 b$ Secondary inputs considered: The following assumptions are made:

(i) Conversion of $R_{k}$ to $E_{k}$ involves $m_{2}$ inputs each of magnitude $F_{j_{2}}\left(j_{2}=1\right.$ to $\left.m_{2}\right)$.

(ii) Conversion of $E_{k}$ to $S_{k}$ involves $m_{1}$ inputs each of magnitude $F_{j_{l}}\left(j_{l}=1\right.$ to $\left.m_{1}\right)$.

Figure 7 gives the resource-task diagram of the centralised energy system with secondary inputs.

The total secondary input is

$$
F=\sum_{k=1}^{p}\left[\sum_{j_{1}=1}^{m_{1}} F_{j_{1} k}+\sum_{j_{2}=1}^{m_{2}} F_{j_{2} k}\right] .
$$

The $T, S, E$ and $R$ values are the same as in $§ 5.2 \mathrm{a}$. The system efficiency is

$$
\eta_{\text {system }}=\left[\sum_{l=1}^{q} T_{l}\right]\left[\sum_{k=1}^{p} S_{k}\right]^{-1}
$$

The overall system efficiency is

$$
\eta_{o \text { system }}=\left[\sum_{l=1}^{q} T_{l}\right]\left[\left(\sum_{k=1}^{p} S_{k}\right)+F\right]^{-1} \text {. }
$$

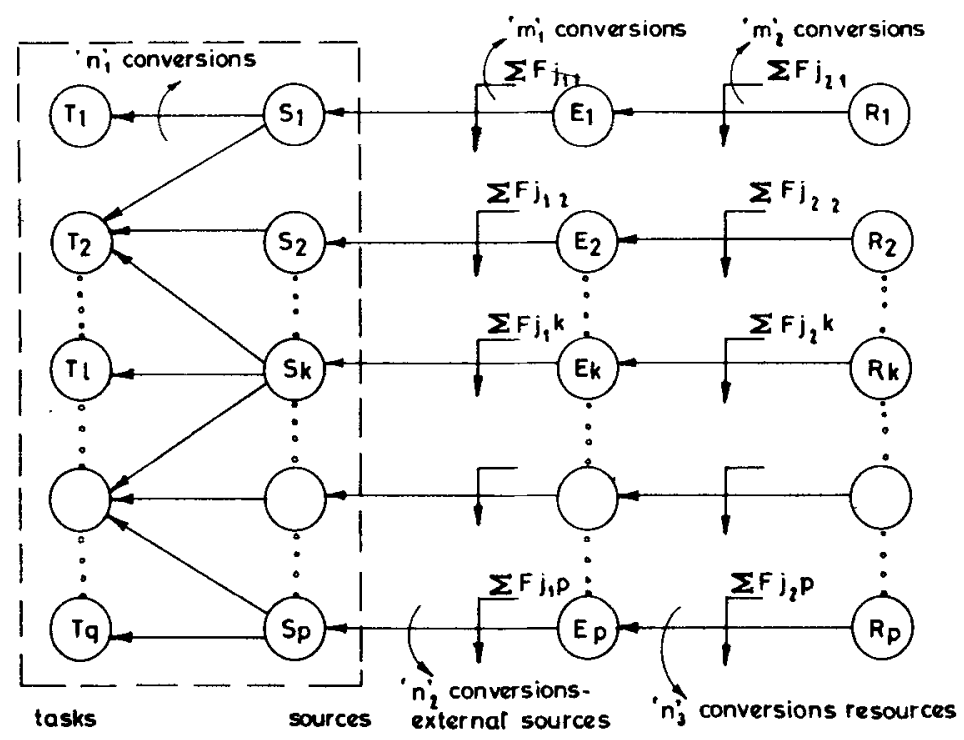

Figure 7. Resource-task diagram of the centralized energy system with secondary inputs $\left(T_{e}\right.$-task energy, $S_{k}$-source energy, $E_{k}$-intermediate energy, $R_{k}-$ resource energy, $F_{\mathrm{j}}$ external energy inputs) 


\subsection{Combined energy system}

The suffix $y$ is used for the decentralised energy components $\left(T_{i y}, S_{l y}\right)$ and the suffix $x$ for the centralised energy components $\left(T_{l x}, S_{k x}, E_{k x}, R_{k x}\right)$.

The following assumptions are made:

(i) There is no internal generation of electricity.

(ii) Tasks using external sources of energy and internally generated sources are quite different and do not overlap.

(iii) Conversion of resource energy $R_{k x}$ to external source $E_{k x}$ involves $m_{2}$ conversions each of overall efficiency $\eta_{i_{2} k x}\left(i_{2}=1\right.$ to $\left.m_{2}\right)$.

(iv) Conversion of external source $E_{k x}$ to village source $S_{k x}$ involves $m_{1}$ conversions each of overall efficiency $\eta_{i_{1} k x}\left(i_{1}=1\right.$ to $\left.m_{1}\right)$.

(v) Conversion of $R_{k x}$ to $E_{k x}$ involves $n_{2}$ inputs each of magnitude $F_{j_{2}}\left(j_{2}=1\right.$ to $\left.n_{2}\right)$.

(vi) Conversion of $E_{k x}$ to $S_{k x}$ involves $n_{1}$ inputs each of magnitude $F_{j_{1}}\left(j_{1}=1\right.$ to $\left.n_{1}\right)$.

(vii) Utilisation of a fraction $\left(S_{k l x}\right)$ of source $S_{k x}$ to perform a fraction $\left(T_{k l x}\right)$ of task $T_{k l}$ involving $m_{1}$ conversions each of overall efficiency $\eta_{i_{x} k l}\left(i_{x}=1\right.$ to $\left.m\right)$.

(viii) Utilisation of a fraction $\left(S_{k l y}\right)$ of source $S_{k y}$ to perform a fraction $\left(T_{k l y}\right)$ of task $T_{k l}$ involving $n$ conversions each of overall efficiency $\eta_{i, k l}\left(i_{y}=1\right.$ to $\left.n\right)$.

Figure 8 gives the resource-task plus the source task diagram of the combined energy system with secondary inputs.

The total source energy using internal sources is

$$
\sum_{k y=1}^{p y} \sum_{l y=1}^{q y} S_{k l y}=\sum_{k y=1}^{p y} \sum_{l y=1}^{q y} T_{l k y}\left[\prod_{i, y=1}^{n} \eta_{i, k l y}\right]^{-1} .
$$

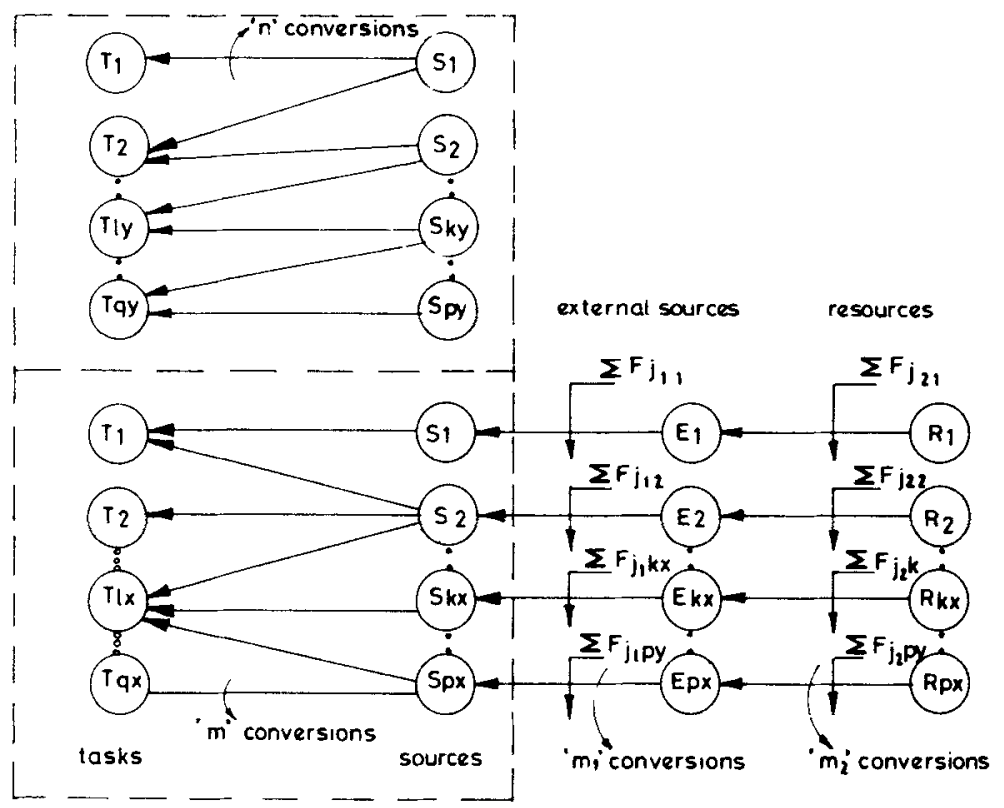

Figure 8. Resource-task + source-task diagram of the combined energy system with secondary inputs $\left(T_{e}\right.$-task energy, $S_{k}$-source energy, $E_{k}$-intermediate energy, $R_{k}$ resource energy, $F_{j}-$ external energy inputs) 
The total source energy using external sources is

$$
\sum_{k x=1}^{p x} \sum_{l x=1}^{q x} S_{k l x}=\sum_{k x=1}^{p x} \sum_{l x=1}^{q x} T_{k i x}\left[\prod_{i_{x}=1}^{m} \eta_{i_{x} l k x}\right]^{-1} .
$$

The total resource energy is

$$
\sum_{k x=1}^{p x} R_{k x}=\sum_{k x=1}^{p x}\left[S_{k x}\left\{\left(\prod_{i_{1}=1}^{m_{1}} \eta_{i_{1} k x}\right)\left(\prod_{i_{2}=1}^{m_{2}} \eta_{i_{2} k x}\right)\right\}^{-1}\right] .
$$

The total secondary input to centralized components is

$$
F=\sum_{k x=1}^{p x}\left[\sum_{j_{1}=1}^{n_{1}} F_{j_{1} k x}+\sum_{j_{2}=1}^{n_{2}} F_{j_{2} k x}\right] \text {. }
$$

The system efficiency is

$$
\begin{aligned}
& \eta_{\text {system }}=\left[\sum_{l=1}^{q} T_{l}\right]\left[\sum_{k=1}^{p} S_{k}\right]^{-1} \\
& =\left[\sum_{l x=1}^{q x} T_{l x}+\sum_{l y=1}^{q y} T_{l y}\right]\left[\sum_{k x=1}^{p x} S_{k x}+\sum_{k y=1}^{p y} S_{k y}\right]^{-1} .
\end{aligned}
$$

The overall system efficiency is

$$
\begin{aligned}
& \eta_{o \text { system }}=\left[\sum_{l x=1}^{q x} T_{l x}+\sum_{l y=1}^{q y} T_{l y}\right] \\
& {\left[\sum_{k x=1}^{p x} R_{k x}+\sum_{k y=1}^{p y} S_{k y}+F\right]^{-1} .}
\end{aligned}
$$

\section{Identification of rural tasks and sources}

By knowing the task end energies from a rural survey, the corresponding source end and resource end energies are determined by using the conversion factors and efficiencies given in this paper. When both the task end and resource end energies are known, the system and the overall system efficiencies are computed.

The first step towards these calculations is to identify rural tasks accurately. These tasks are classified into three groups.

(i) Type I (human and animal powered tasks): These comprise a large variety of tasks which are entirely different from each other and are performed sequentially. Replacement of these by mechanisation involves special machines whose operating time is low and expenditure is enormous. Thus these tasks are essentially not substitutable by other energy forms.

(ii) Type II (human and animal powered tasks): These are a small number of highly repetitive jobs - that is, repetition of the same type of motion a large number of times, $e g$, pumping water, hand pounding of rice, extraction of oil from coconuts, etc. These tasks can be mechanised.

(iii) Type III (fueled by non-living sources of energy): This includes a large set of tasks such as cooking by firewood, lighting by electricity, etc.

Table 1 gives different sources and tasks and also the present and possible future 


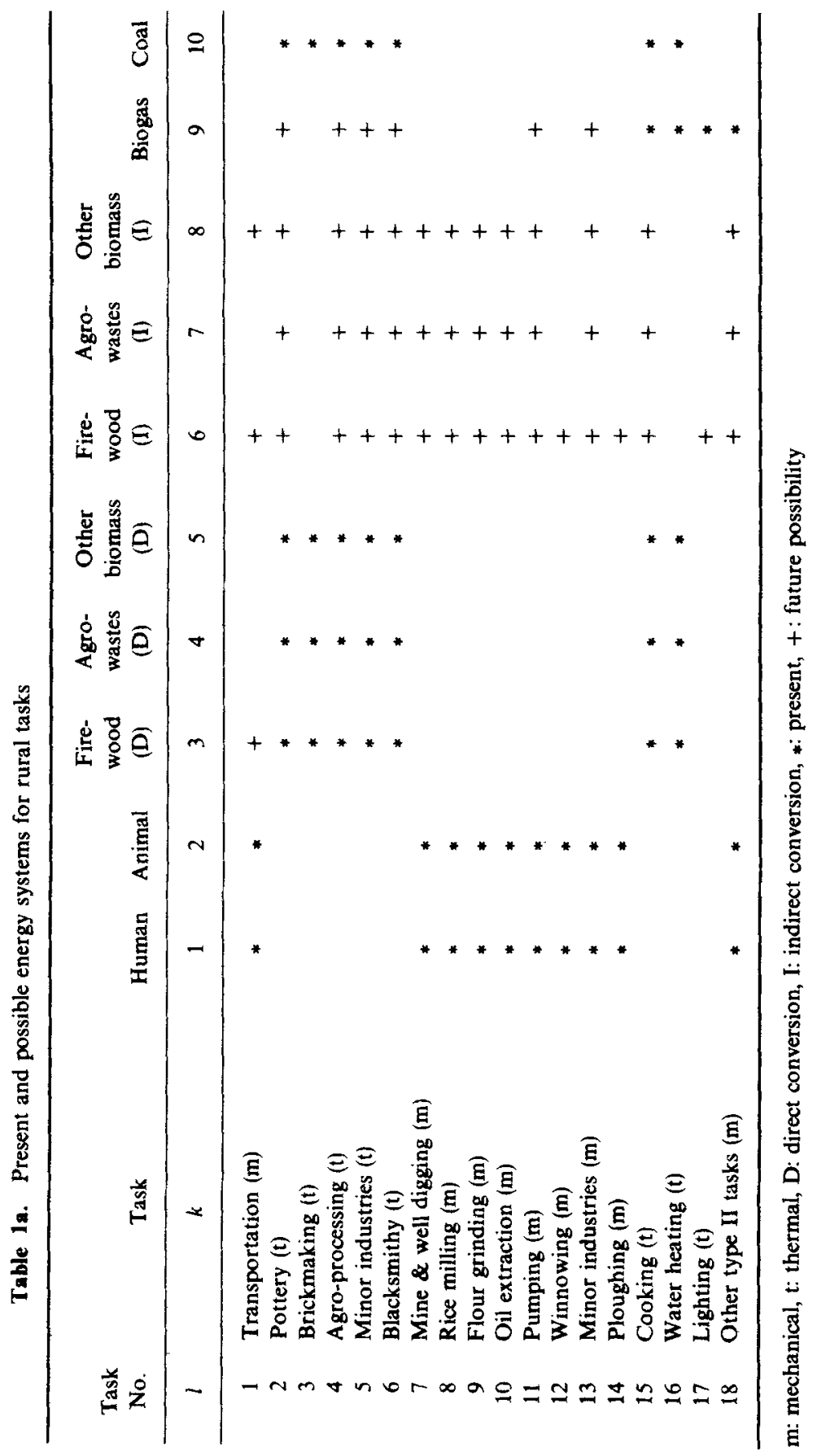




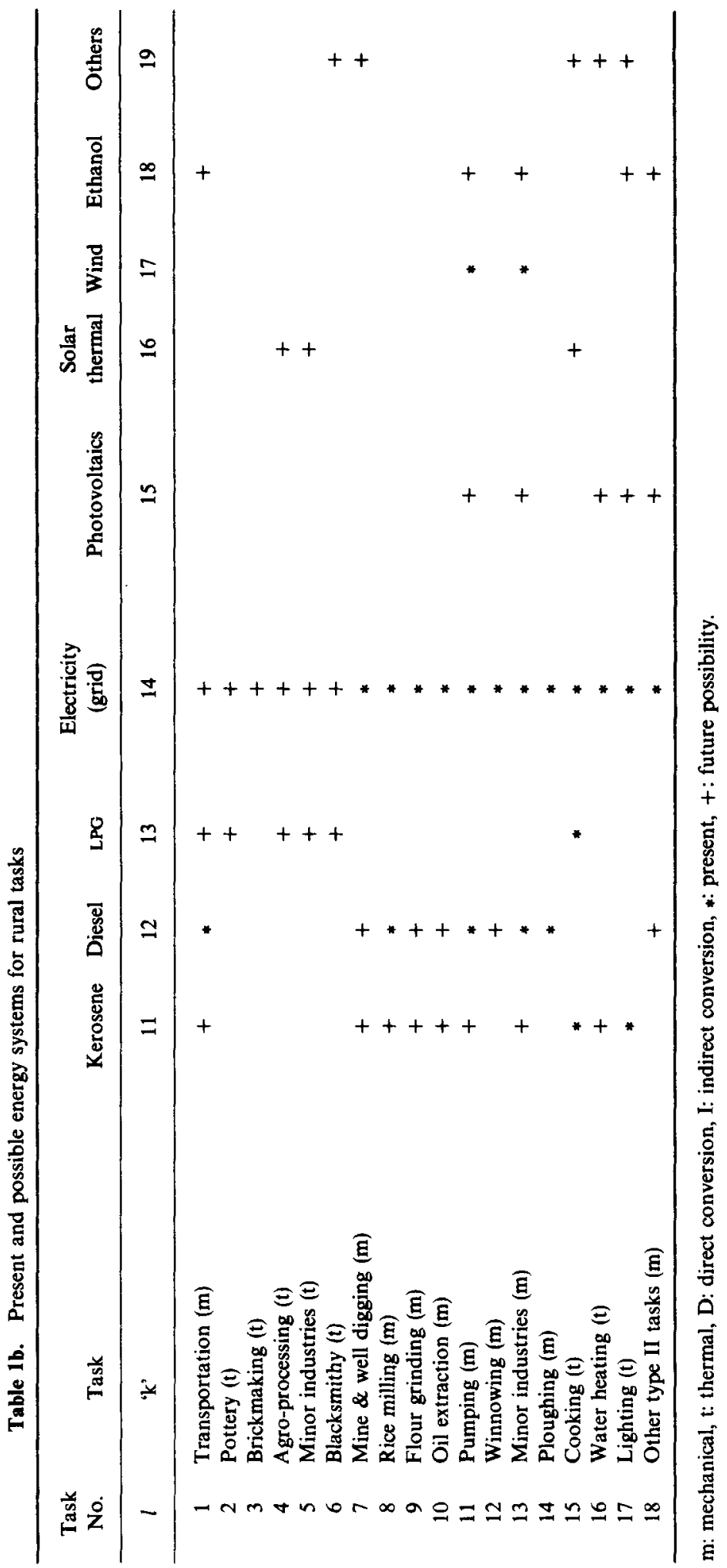


energy systems for rural tasks. Direct denotes direct combustion and indirect denotes usage after conversion into secondary fuels. Other biomass denotes casuarina loppings, groundnut shells, toddy leaves, etc., Firewood (indirect) includes conversion to charcoal, pyrolytic oil, producer gas, etc. Agrowastes (indirect) include conversion to char briquettes, briquettized fuel, pellets, producer gas, etc. Other biomass (indirect) refers to briquettes, pellets, producer gas, etc, from thermal and mechanical processes. It also includes wastes which can be incinerated to yield heat. Biogas is from animal dung, human refuse, kitchen waste, water hyacinth and other non-woody biomass.

\section{Typical tasks for a large village}

In order to get an idea of the magnitude of rural loads, the task end energy is computed from a survey of Muthukur village (population 10,000) in the Nellore District of Andhra Pradesh. The survey was conducted by the author during April-May 1982. Attached to the village are 6896 acres of paddy ( $80 \%$ with 2 crops/year), 415 acres of

Table 2. Energy loads in Muthukur Village on a yearly basis

\begin{tabular}{|c|c|c|c|c|c|}
\hline $\begin{array}{c}\text { Task } \\
\text { Code } \\
\text { No. }\end{array}$ & Device & $\begin{array}{c}P_{\mathbf{0}} \\
(\mathrm{kW})\end{array}$ & $\begin{array}{c}N \\
(-)\end{array}$ & $\begin{array}{c}\tau \\
(h)\end{array}$ & $\begin{array}{c}E_{0} \\
\left(\times 10^{6} \mathrm{kcal}\right)\end{array}$ \\
\hline 1 & Labourer (type-II tasks) $^{+}$ & 0.08 & 4600 & 2400 & $759 \cdot 6$ \\
\hline 2 & Cattle (type-1I tasks) ${ }^{++}$ & 0.36 & 600 & 1200 & $222 \cdot 9$ \\
\hline 3 & Bullock cart & 0.72 & 674 & 1200 & 500.8 \\
\hline 4 & Ploughing force & 0.80 & 1021 & 104 & 73.00 \\
\hline 5 & Domestic cookstove & 0.80 & 2072 & 1830 & $2608 \cdot 7$ \\
\hline 6 & Hotel cookstove & $1 \cdot 20$ & 2 & 2700 & 5.6 \\
\hline 7 & Bathwater heater & 1.40 & 1288 & 480 & $744 \cdot 4$ \\
\hline 8 & Baker's oven & $2 \cdot 0$ & 1 & 500 & 0.9 \\
\hline 9 & Potter's kiln & $18 \cdot 0$ & 1 & 72 & $1 \cdot 1$ \\
\hline 10 & Brick burning kiln & $45 \cdot 0$ & 1 & 2200 & $85 \cdot 1$ \\
\hline 11 & Blacksmith's furnace & 1.67 & 1 & 2400 & 3.4 \\
\hline 12 & Tobacco curing kiln & $4 \cdot 20$ & 1 & 125 & 0.5 \\
\hline 13 & Oil extracting mill & 3.73 & 1 & 600 & 1.9 \\
\hline 14 & Diesel pumpset & 5.97 & 157 & 340 & $274 \cdot 1$ \\
\hline 15 & Rice mill & $33 \cdot 57$ & 1 & 4620 & 133.4 \\
\hline 16 & Flour mill & 3.73 & 1 & 4500 & $14 \cdot 4$ \\
\hline 17 & Small kerosene lamp & 0.0015 & 2327 & 1095 & $3 \cdot 3$ \\
\hline 18 & Big kerosene lamp & 0.003 & 2215 & 1450 & $8 \cdot 3$ \\
\hline 19 & Domestic electric light & 0.04 & 2306 & 1095 & 86.9 \\
\hline 20 & Fan & $0 \cdot 10$ & 291 & 730 & $18 \cdot 3$ \\
\hline \multirow[t]{2}{*}{21} & Street light & & & & \\
\hline & (Incandescent) & $0 \cdot 10$ & 397 & 1825 & $62 \cdot 3$ \\
\hline 22 & Electric pumpset & 3.73 & 846 & 290 & $787 \cdot 0$ \\
\hline 23 & Tractor & $26 \cdot 11$ & 30 & 203 & $136 \cdot 7$ \\
\hline 24 & Truck & $104 \cdot 4$ & 1 & 800 & $71 \cdot 8$ \\
\hline 25 & Bus & $89 \cdot 5$ & 1 & 68 & $5 \cdot 3$ \\
\hline
\end{tabular}

Note: ${ }^{+}$This includes energy for minedigging $-0.03 \times 10^{6} \mathrm{kcal} ;{ }^{++}$Other than ploughing and carting; $P_{0}$ : Power output; $N$ : Number of devices; $\tau$ : operating time/device/year, $E_{0}=$ energy output/year $=\left(P_{0} N \tau\right)$ 
sugarcane, 2255 acres of groundnut, 327 acres of casuarina firewood plantation, 85 acres of eucalyptus and 120 acres of chilli plantations. There are nearly 1200 acres of grazing and 500 acres of waste land and 9500 head of cattle. Table 2 gives the energy loads in Muthukur village on a yearly basis.

In devices where the power output varies widely, the average power output is considered (total energy output in one operation/operating time). In rated devices, the most common value is chosen. When the power outputs are different from the values shown in the table, the number of devices equivalent to the rated power are considered, $e g$, if the average power of lamps is $40 \mathrm{~W}$ and if there is a $1 \mathrm{~kW}$ lamp it is taken as 25 lamps of $40 \mathrm{~W}$. The minor efficiency variations from this change are neglected. When operating time varies, the average number of hours of operation of the standard device is considered, eg, if three farmers having $5 \mathrm{HP}(3.73 \mathrm{~kW})$ pumpsets operate them for $300,600,500 \mathrm{hr} /$ year, the operating time is taken as $467 \mathrm{hr} /$ year/pump.

\section{Overall efficiencies used for calculations}

Tables 3 to 7 give the overall efficiencies of rural devices for various tasks. The overall efficiency in this constant context is defined as, energy output of device-energy input to device in the form of the given source, for a particular time interval. The efficiencies of rural devices and tasks used in tables 3 to 6 have been computed by Bhatt \& Reddy $(1982,1983,1984)$. The overall system efficiencies in table 7 have been computed by Bhatt (1984).

The values of the overall efficiencies of rural devices are calculated from efficiencies of individual devices. Some of the useful values are:

Diesel engine: $40 \%$; ethanol engine: $30 \%$; producer gas engine: $30 \%$; biogas engine: $30 \%$; fuel cell: $70 \%$; lead acid battery: 73-87\%; steam engine: $20-24 \%$; steam turbine: $35 \%$; gas turbine: $30 \%$; windmill: 6-18\%; water wheel: $65 \%$; solar cell: $13 \%$; wood gasifier: $78 \%$; solar flat plate collector: $50 \%$; solar drier: $55 \%$; thermal power plant: 16-25\%; electric generator: $80-90 \%$; electric motor: $75-85 \%$; electromechanical coupling: $90-98 \%$.

Some of these efficiencies are dependent on the size of the device. For the purpose of calculations, normal sizes suited for rural use are considered.

Table 3. Overall system efficiencies--transportation

\begin{tabular}{lccc}
\hline Sources & $\begin{array}{c}\text { Transportation } \\
\text { (human) }\end{array}$ & $\begin{array}{c}\text { Transportation } \\
\text { (goods) }\end{array}$ & Ploughing \\
\hline Human & $13^{(1)}$ & $5^{(2)}$ & $3^{(3)}$ \\
Bullocks & $5^{(4)}$ & $5^{(4)}$ & $5^{(5)}$ \\
Diesel & 9 & 9 & 18 \\
Agrowastes (fuel cells) & 18 & 18 & 30 \\
Firewood (producer gas) & 6 & 6 & 13 \\
Firewood (pyrolytic oil) & 7 & 7 & 14 \\
Ethanol & 9 & 9 & 18 \\
Electricity (lead acid batteries) & 18 & 18 & 32 \\
\hline
\end{tabular}

1: bicycle; 2: hand drawn cart; 3: hand drawn plough; 4: carts; 5: bullock drawn plough. The other values in the three columns denote efficiencies of bus, truck and tractor respectively. 


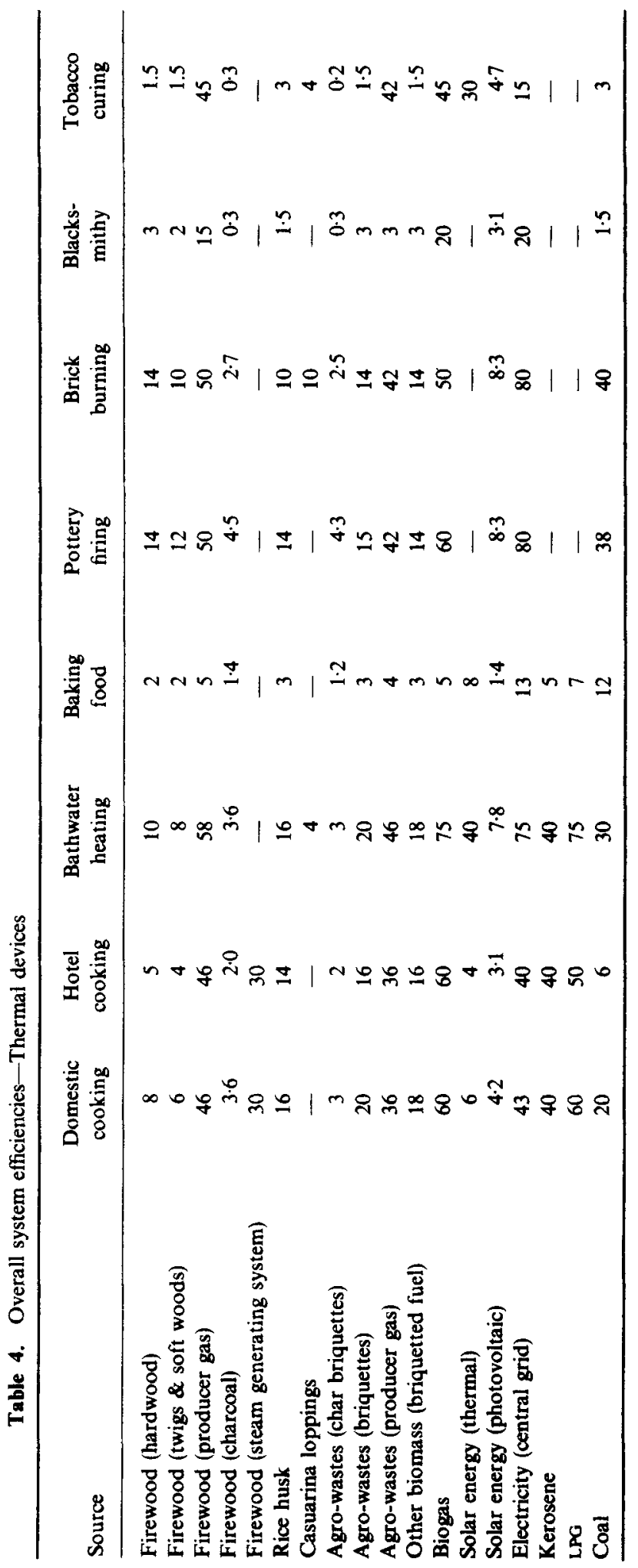


Table 5. Overall system efficiencies-mechanical power devices

\begin{tabular}{|c|c|c|c|c|}
\hline Sources & $\begin{array}{c}\text { Oil } \\
\text { extraction }\end{array}$ & $\begin{array}{c}\text { Water } \\
\text { pumping }\end{array}$ & $\begin{array}{c}\text { Rice } \\
\text { milling }\end{array}$ & $\begin{array}{l}\text { Flour } \\
\text { grinding }\end{array}$ \\
\hline Human power & 3 & 3 & 3 & 3 \\
\hline Bullock power & 5 & 5 & 5 & 5 \\
\hline Firewood (producer gas engine) & 23 & 19 & 24 & 24 \\
\hline Agro-wastes (producer gas engine) & 21 & 17 & 21 & 21 \\
\hline Other biomass (producer gas engine) & 23 & 19 & 24 & 24 \\
\hline Biogas (IC engine) & 32 & 30 & 32 & 32 \\
\hline Diesel (IC engine) & 28 & 24 & 30 & 30 \\
\hline Ethanol (IC engine) & 24 & 20 & 24 & 24 \\
\hline Electricity (conventional grid) & 30 & 30 & 32 & 28 \\
\hline Solar photovoltaics (electric motor) & $3 \cdot 5$ & $3 \cdot 5$ & $3 \cdot 7$ & $3 \cdot 3$ \\
\hline Solar thermal (thermodynamic cycle) & $0 \cdot 1$ & 0.1 & 0.1 & $0 \cdot 1$ \\
\hline $\begin{array}{l}\text { Windmill (mechanical coupling with energy } \\
\text { storage capacity) }\end{array}$ & 4 & 6 & 3 & 3 \\
\hline
\end{tabular}

IC-Indirect combustion

Table 6. Overall system efficiencies-fan and lighting

\begin{tabular}{lccc}
\hline Source & $\begin{array}{c}\text { Domestic } \\
\text { fan power }\end{array}$ & $\begin{array}{c}\text { Domestic } \\
\text { lighting }\end{array}$ & $\begin{array}{c}\text { Street } \\
\text { lighting }\end{array}$ \\
\hline Human power (dynamo with fan/bulb) & $2 \cdot 5$ & 0.04 & 0.04 \\
Animal power (dynamo with fan/bulb) & 34 & 0.05 & 0.05 \\
Firewood (pyrolytic oil lamp) & - & 0.5 & 0.3 \\
Firewood (natural oil lamp) & - & 0.8 & 0.5 \\
Biogas (mantle) & - & 14 & 12 \\
Ethanol (lantern) & - & 0.5 & $0 \cdot 3$ \\
Kerosene (lantern/pressure lamp) & - & 0.01 & 0.01 \\
Electricity from central grid (bulb/fan) & 0.75 & $1 \cdot 5^{+}$ & $1 \cdot 5^{+}$ \\
Solar photovoltaic with storage (fan/bulb) & 7 & $(6)^{*}$ & $(6)^{*}$ \\
& & $(0.16)^{+}$ & $(0.16)^{+}$ \\
\hline
\end{tabular}

+ Incandescent lamp, * Fluorescent lamp

Table 8 gives the secondary energy inputs associated with sources external to the village. These values have been computed by Bhatt (1984).

\section{Results}

\subsection{Common energy data (decentralized system)}

9.1 One-source model: The overall system efficiency (\%) for the tasks in table 2 are as follows:

Source-firewood: $3 \cdot 15$; solar photovoltaics: $2 \cdot 92$; rice husk: $2 \cdot 70$; biogas: $5 \cdot 40$. 


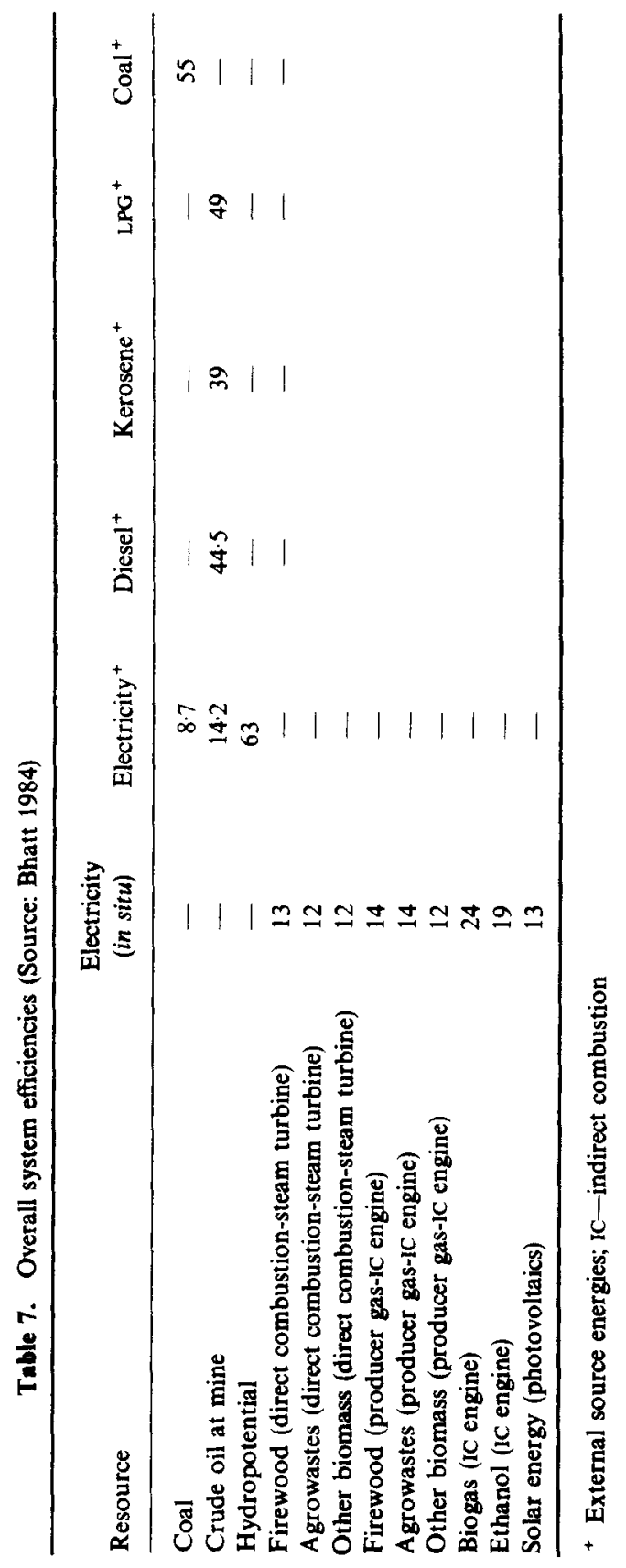


Table 8. Secondary energy inputs associated with sources external to the village.

\begin{tabular}{lcc}
\hline Source $^{+}(\mathbf{k})$ & $\begin{array}{c}\sum_{j_{1}=1}^{m_{1}} F_{j_{1} k} \\
\left(\times 10^{3} \mathrm{kcal}\right)\end{array}$ & $\begin{array}{c}\sum_{j_{2}=1}^{m_{2}} F_{j_{2} k} \\
\left(\times 10^{3} \mathrm{kcal}\right)\end{array}$ \\
\hline Electricity (MWh) & $793 \cdot 1$ & - \\
LPG (t) & $318 \cdot 3$ & $1212 \cdot 3$ \\
Kerosene (t) & $510 \cdot 8$ & $508 \cdot 7$ \\
Diesel (t) & 447.6 & $445 \cdot 8$ \\
Coal (t) & $404 \cdot 5$ & $178 \cdot 2$ \\
\hline
\end{tabular}

+ at village source end

9.1b Multi-source model: The following sources are assumed (percentages at the source end): firewood-70\%; solar photovoltaics $-12.5 \%$; agrowastes $-5 \%$; biogas$12.5 \%$.

The overall system efficiency for the above tasks is $3 \cdot 25 \%$.

\subsection{One energy mode}

If firewood is assumed to be the single energy source the overall system efficiency is $6.92 \%$.

\subsection{Multi-energy mode}

The percentages of different sources at the source end are: firewood- $5 \cdot 7$; agrowastes9.6; biogas-11.6; animal energy -22.5; human energy-44.2; solar energy -0.003 ; ethanol-1.3. The overall system efficiency is $11.28 \%$.

9.4a Complex energy mode: The source energy for electrical tasks is solar photovoltaics, rice husk and firewood; and for non-electrical tasks is firewood, biogas, ethanol, rice husk and solar energy. The overall system efficiency is $7.05 \%$.

9.4b Centralized systems: The energy sources are electricity, diesel, coal, kerosene and LPG. The overall system efficiency and the system efficiency considering secondary inputs are 4.06 and $29.80 \%$, and without considering them are 4.09 and $29.98 \%$ respectively.

9.4c Combined energy system: The energy source is: non-electrical decentralized components-biogas and rice husk; decentralized electricity producing componentfirewood; centralized components-electricity and diesel. The overall system efficiency and the system efficiency are $5 \cdot 71$ and $7.89 \%$ respectively.

\section{Discussions and conclusions}

(i) This paper gives the methodology together with the relevant data for finding out the energy consumption of different rural energy systems. Given the tasks, the energy resources needed for sustaining these can be determined. In the Indian context, no single energy strategy is suited for all conditions. This methodology enables one to find 
out the resource energy for any giver, set of tasks considering the peculiarities of the region. From a knowledge of the current task end energy, the task energy needed ten or twenty years hence can be estimated by forecasting techniques (not discussed here), and from this estimate the energy resources from local and centralized sources needed for the future can be calculated.

It must be mentioned here that this methodology is not an optimisation technique nor does it take economics into consideration; but by using various combinations of sources, tasks, devices and routes, one can arrive at a combination offering high efficiency within the resource and economic constraints.

(ii) The energy problem is so serious that heavy reliance on any one energy form, eg, firewood or electricity, will not help. Diversification through multiple source systems has to be reckoned as the only solution to the problem. Multiple source systems involve interaction with various energy departments like the forest departments, electricity boards, thermal or hydropower corporations, etc. This is possible only if there is a framework of the multiple energy system where the resources, sources, tasks, devices and routes are clearly known. The current method enables one to choose the fractions of different resources to be developed for a village so as to energise all tasks in any time horizon. The choice is made by finding out the resource or source end energies for a given set of tasks assuming that they are energised by different energy resources.

(iii) The share of centralised energy to a village could be modified according to its local resource potential. By knowing the available resources (local), the tasks and routes, the decentralised components can be determined. Only the balance need be supplied by the centralised sources.

(iv) The method enables one to have an idea of the magnitudes of the overall system efficiencies of the present day rural energy systems in developing countries. For various other routes, the overall system efficiencies can be determined and improvements if any with them can be compared with the economic inputs needed to install the new systems. (v) A look at the different energy conversion routes for the same source performing the same tasks suggests ways of conserving energy, eg, firewood for domestic cooking through the charcoal route gives $3.6 \%$ efficiency whereas through the producer gas pathway gives $46 \%$.

(vi) It is also possible to evaluate the energy conservation that can be achieved by a particular scheme so that its worthiness can be tested, eg, electrical energy savings in a model village by the replacement of all existing incandescent lamps by fluorescent tubes.

(vii) The multi-energy mode of the decentralized system is the most efficient system as its efficiency is the highest $(11.28 \%)$. This is the ideal system for villages.

(viii) Common energy models (electricity, hydrogen, methane, etc.) are infeasible because of low efficiencies and high costs. One energy source models are also infeasible because the energy resources required are nearly ten times more than the village production.

(ix) Centralized systems have low overall system efficiencies but high system efficiencies $(<30 \%)$. It is not advisable to utilise the scarce fossil fuels at such low efficiencies. Instead, local alternatives could be developed.

The author wishes to thank the referee for the excellent review which has resulted in the rewriting of the paper in its present form. He also wishes to thank Prof. Roddam Narasimha for valuable suggestions. 


\section{References}

Bhatt M S, Reddy B S 1982 Changing villages 4: 1-37

Bhatt M S, Reddy B S 1983 Res. Ind. 28: 12-24

Bhatt M S, Reddy B S 1984 Energ. manage. (India) 8: 113-121

Bhatt M S 1984 Ind. Eng. J. 13: 8-16 